\title{
ON THE ORBITAL MOTION DESCRIPTION WITH A PROPER REFERENCE FRAME IN A COMPLETE SCHWARZSCHILD FIELD
}

\author{
J.M. GAMBI AND P. ZAMORANO
}

Departamento de Matemáticas. Escuela Politécnica Superior. Universidad Carlos III. Madrid.

\section{AND \\ P. ROMERO AND M.L. GARCIA DEL PINO}

Sección Departamental de Astronomía y Geodesia. Facultad de Ciencias Matemáticas. Universidad Complutense. Madrid.

It is a fact that, none of the diverse coordinate systems unified in the Post-Newtonian formalism used to describe the exterior Schwarzschild field can be regarded as being materialized by a reference frame. Only the polar Gaussian coordinates $(\rho, \vartheta, \varphi, t)$, or their naturally associated Fermi coordinates, can be shown to have this property (Synge, 1960).

As it is known, each one of them has a precise physical meaning and by means of them at least four new results appear.

In effect, by using the general expression for the world function (Synge, 1960 ) in these coordinates, it is easy to see that for an observer at rest with respect to the Sun, the relativistic distance $\left|\rho_{1}-\rho_{2}\right|$, between two particles aligned with him, becomes their Euclidean distance; this is the first result.

Now, starting from the lagrangian $L$, written again in these coordinates, corresponding to a test particle moving in the exterior field, and using the first integrals in the usual way, the equation of the trajectory $\left(\vartheta=\pi / 2, \vartheta^{\prime}=0\right)$ results to be

$$
\begin{aligned}
\left(\frac{d u}{d \varphi}\right)^{2} & =f(u)=\alpha^{2}\left(\beta^{2}-1\right)+2 m \alpha^{2} \beta^{2} u+4 m \alpha^{2}\left(\beta^{2}-1\right) u \log u+ \\
& +\left(-1+4 m^{2} \alpha^{2}\right) u^{2}+6 m^{2} \alpha^{2} \beta^{2} u^{2} \log u-2 m u^{3} \log u
\end{aligned}
$$


where $u=1 / \rho, \alpha^{-1}=\frac{\partial L}{\partial \varphi^{\prime}}$ and $-\beta=\frac{\partial L}{\partial t^{\prime}}$ so that, as can be seen, the right side in (1) is not a cubic as it happens to be when the standard coordinates, for example, are used. This is the second result.

Nevertheless, it can be shown that not even being $f(u)$ a cubic (it can be shown that it has only two roots) the solution of (1) can be given in terms of elliptic functions. This is the third result.

Finally, the fourth result may help to better understand some properties in Orbital Dynamics and Astrometry: as it is known, for coordinates of a static weak gravitational field, for which the metric is written as $\eta_{i j}+h_{i j}$, the equations of motion for a test particle in first approximation contain the terms

$$
\frac{1}{2} h_{44, \alpha} \text { and } \quad-\frac{1}{2} h_{\alpha \beta} h_{44, \beta}
$$

(Brumberg, 1991).The second term comes from the unique non-linear term taken in the Christoffel symbols and is shown to play an important role as a source of relativistic perturbations. For example, in standard coordinates, this term appears as the first one in the right hand side of the equations

$$
\ddot{\vec{r}}+\frac{m}{r^{3}} \vec{r}=\frac{m}{r^{3}}\left[\left(\frac{2 m}{r}-2 \dot{\vec{r}}^{2}+3 \frac{(\dot{\vec{r} \overrightarrow{\vec{r}}})^{2}}{r^{2}}\right) \vec{r}+2(\dot{\vec{r}}) \dot{\vec{r}}\right]
$$

But, if polar Gaussian coordinates are used, we obtain

$$
\begin{aligned}
\ddot{\vec{\rho}}+ & \left(\frac{m}{\rho^{3}}-\frac{m^{2}}{\rho^{4}}(1-2 \log \rho)\right) \vec{\rho}=\frac{m}{\rho^{3}}\left[\left(-(1+\log \rho) \dot{\vec{\rho}}-\frac{(1-3 \log \rho)}{\rho^{2}} .\right.\right. \\
& \left.\left.\cdot(\vec{\rho} \dot{\vec{\rho}})^{2}\right) \vec{\rho}+\left(2(2-\log \rho)-\frac{2 m}{\rho}(1-2 \log \rho)\right)(\dot{\vec{\rho}} \dot{\vec{\rho}}) \dot{\vec{\rho}}\right]
\end{aligned}
$$

so that the analogous to this term in (3) is now the third one in the left hand side of (4) and although, obviously, their effects in the perturbed motion are analogous (but not equal in any way, as can be imagined from the previous results) an important difference between their origins emerges, and this is that, whereas the cited term in (3) comes from the second one in (2) as was said, the cited term in (4) comes from the first one in (2), so that no non-linear term is needed to be introduced in the Christoffel symbols to explain the perturbations when polar Gaussian (or Fermi) coordinates are used. In fact, if the second term in (2) is evaluated in these coordinates the result is that its value is, simply, zero.

\section{References}

Synge,J.L., Relativity: The General Theory. North Holland. N.Y., 1960.

Brumberg,V.A., Essential Relativistic Celestial Mechanics. Adam Hilger, N.Y., 1991. 